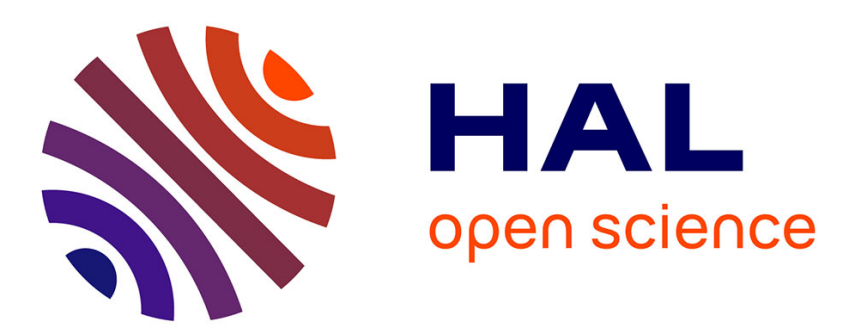

\title{
On the closed loop identification of LPV models using instrumental variables
}

Roland Toth, Vincent Laurain, Marion Gilson, Hugues Garnier

\section{To cite this version:}

Roland Toth, Vincent Laurain, Marion Gilson, Hugues Garnier. On the closed loop identification of LPV models using instrumental variables. 18th IFAC World Congress, IFAC WC'2011, Aug 2011, Milan, Italy. pp.7773-7778. hal-00654796

\section{HAL Id: hal-00654796 https://hal.science/hal-00654796}

Submitted on 22 Dec 2011

HAL is a multi-disciplinary open access archive for the deposit and dissemination of scientific research documents, whether they are published or not. The documents may come from teaching and research institutions in France or abroad, or from public or private research centers.
L'archive ouverte pluridisciplinaire $\mathbf{H A L}$, est destinée au dépôt et à la diffusion de documents scientifiques de niveau recherche, publiés ou non, émanant des établissements d'enseignement et de recherche français ou étrangers, des laboratoires publics ou privés. 


\title{
On the closed loop identification of LPV models using instrumental variables
}

\author{
Roland Tóth* Vincent Laurain ${ }^{* *}$ Marion Gilson** \\ Hugues Garnier** \\ * Delft Center for Systems and Control, Delft University of \\ Technology, Mekelweg 2, 2628 CD, Delft, The Netherlands (e-mail: \\ r.toth@tudelft.nl) \\ ** Centre de Recherche en Automatique de Nancy (CRAN), \\ Nancy-Université, CNRS, BP 70239, 54506 Vandoeuvre-les-Nancy \\ Cedex, France (e-mail: \{vincent.laurain, marion.gilson, \\ hugues.garnier\}@cran.uhp-nancy.fr.)
}

\begin{abstract}
Identification of real life systems is often applied in closed-loop due to stability, performance or safety constraints. However, in case of Linear Parameter-Varying (LPV) systems, closed-loop identification is not well-established despite the recent advances in prediction-error identification. Building on the available results, the paper proposes the closed-loop generalization of a recently introduced instrumental variable scheme for the identification of LPV-IO models with Box-Jenkins type of noise models. Estimation under closed-loop conditions is analyzed from the stochastic point of view with the proposed approach and the performance of the method is demonstrated through a representative simulation example.
\end{abstract}

Keywords: LPV models, system identification, Refined Instrumental Variable, Box--Jenkins models, Input/Output, Transfer function

\section{INTRODUCTION}

Identification of physical or chemical systems is often restricted to data gathered during closed-loop operation due to stability, performance/economical or safety constraints. The fact that the control loop itself introduces correlation between the disturbances and the control signal yields that the statistically optimal estimation of the parameters of a chosen model structure w.r.t. the data-generating system is an essentially different problem than in the open-loop setting. Hence in the identification literature, especially in the linear time-invariant (LTI) case, many approaches have been proposed to provide well-applicable solutions in this problem setting. An overview of the developments in the LTI case can be found in Van den Hof (1998) and Forssell and Ljung (1999).

Identification of linear parameter-varying (LPV) systems has recently seen significant improvements in terms of open-loop methods, however closed loop-identification has remained sparsely studied (see Casella and Lovera (2008); Tóth $(2010)$ ). The main difficulty in this system class in terms of identification is that even if the signal relations between the inputs $u$ and outputs $y$ of the system are linear, these relations are allowed to change over time as a function of a so called scheduling variable $p: \mathbb{Z} \rightarrow \mathbb{P}$ with $\mathbb{P} \subseteq \mathbb{R}^{n_{\mathrm{p}}}$ being the so called scheduling space. This allows to describe a large class of nonlinear/time-varying systems in an attractive structure allowing the use of well-established LPV control-synthesis approaches, e.g.

\footnotetext{
* Supported by the Netherlands Organization for Scientific Research (grant no. 680-50-0927).
}

Packard (1994); Scherer (1996). On the other hand, this varying-relation prevents the use of crucial system theoretical relations, like transfer functions and commutativity of operators, which are often used in LTI closed-loop identification approaches. As a consequence, only preliminary closed-loop approaches has been proposed in the literature without being able to exploit the existing tools and knowledge available in the LTI case. In van Wingerden and Verhaegen (2009), an approximation based LPV extension of a predictor subspace approach (PSBID) has been proposed which is also applicable in a closed-loop setting, while in Boonto and Werner (2008) also an approximation based LPV extension of the CLOE algorithm (see Landau and Karimi (1997)) has been investigated w.r.t. LPV outputerror (OE) type of models. In Abbas and Werner (2009) and Butcher et al. (2008) a basic instrumental variable method has been introduced. It is a clear indicator of the immature state of this research direction that the stochastic properties of the estimation has been analyzed only in Butcher et al. (2008). Unfortunately in Laurain et al. (2010), it has been shown recently that the formulation of the one-step IV approach proposed in Butcher et al. (2008) does not allow to reach statistically optimal estimates due no-commutativity of certain filtering operations. This highlights that currently no method has been established which allows stochastically optimal estimation under a closed-loop setting especially with general noise models like Box-Jenkins (BJ).

Based upon the available results of Abbas and Werner (2009); Butcher et al. (2008) and using the recent theoretical advancements of the LPV prediction error-framework 
introduced in Tóth (2010), we propose in this paper a closed-loop extension of a recently developed IV approach for LPV-BJ models, see Laurain et al. (2010). In this setting, the noise $v_{\mathrm{o}}$ affecting the sampled output measurement $y\left(t_{k}\right)$ is assumed to have a rational spectral density which is not restricted to depend on the actual process dynamics of the data-generating system (general noise structure). As a first step towards the case of a $p$ dependent noise, it is also assumed that this rational spectral density does not depend on $p$. A stochastic analysis of the proposed closed-loop approach is provided, exploring the limitations and the benefits of this estimation strategy. The performance of the algorithm is also demonstrated on a representative simulation example.

\section{PROBLEM DESCRIPTION}

\subsection{System description}

Consider the data-generating LPV system $\mathcal{S}_{\mathrm{o}}$ given in a closed-loop setting of Fig. 1 , where $\mathcal{S}_{\mathrm{o}}$ is defined in the following form:

$$
\begin{aligned}
A_{\mathrm{o}}\left(p_{k}, q^{-1}\right) \chi_{\mathrm{o}}\left(t_{k}\right) & =B_{\mathrm{o}}\left(p_{k}, q^{-1}\right) u\left(t_{k-d}\right), \\
y\left(t_{k}\right) & =\chi_{\mathrm{o}}\left(t_{k}\right)+v_{\mathrm{o}}\left(t_{k}\right),
\end{aligned}
$$

Here $u\left(t_{k}\right)$ is the input of the plant, $p_{k}$ is the value of the scheduling variable $p$ at sample time $t_{k}, \chi_{\mathrm{o}}$ is the noisefree output, $v_{\mathrm{o}}$ is the additive noise with bounded spectral density, $y$ is the noisy output of the system, $d$ is the delay, and $q$ is the time-shift operator, i.e. $q^{-i} u\left(t_{k}\right)=u\left(t_{k-i}\right)$. $A_{\mathrm{o}}\left(p_{k}, q^{-1}\right)$ and $B_{\mathrm{o}}\left(p_{k}, q^{-1}\right)$ are polynomials in $q^{-1}$ of degree $n_{\mathrm{a}}$ and $n_{\mathrm{b}}$ respectively:

$$
\begin{aligned}
& A_{\mathrm{o}}\left(p_{k}, q^{-1}\right)=1+\sum_{i=1}^{n_{\mathrm{a}}} a_{i}^{\mathrm{o}}\left(p_{k}\right) q^{-i} \\
& B_{\mathrm{o}}\left(p_{k}, q^{-1}\right)=\sum_{j=0}^{n_{\mathrm{b}}} b_{j}^{\mathrm{o}}\left(p_{k}\right) q^{-j} .
\end{aligned}
$$

Here the coefficients $a_{i}$ and $b_{j}$ are real meromorphic functions ${ }^{1}$ with static dependence on $p$, i.e. dependence only on the instantaneous value of $p$ at time $t_{k}$. It is assumed that each $a_{i}$ and $b_{j}$ is non-singular on $\mathbb{P}$, thus the solutions of $\mathcal{S}_{\mathrm{o}}$ are well-defined and the process part $\mathcal{G}_{\mathrm{o}}$, defined by (1a), is completely characterized by the coefficient functions $\left\{a_{i}^{\mathrm{o}}\right\}_{i=1}^{n_{\mathrm{a}}}$ and $\left\{b_{j}^{\mathrm{o}}\right\}_{j=0}^{n_{\mathrm{b}}}$.

For the sake of simplicity, the noise $v_{\mathrm{o}}$ is assumed to be independent from $p$. In case of rational spectral density of $v_{\mathrm{O}}$, such a noise process is represented by a discrete-time auto-regressive moving average (ARMA) model:

$$
v_{\mathrm{o}}\left(t_{k}\right)=H_{\mathrm{o}}(q) e_{\mathrm{o}}\left(t_{k}\right)=\frac{C_{\mathrm{o}}\left(q^{-1}\right)}{D_{\mathrm{o}}\left(q^{-1}\right)} e_{\mathrm{o}}\left(t_{k}\right),
$$

where $C_{\mathrm{o}}\left(q^{-1}\right)$ and $D_{\mathrm{o}}\left(q^{-1}\right) \neq 0$ are monic polynomials with constant coefficients and with respective degree $n_{\mathrm{c}}$ and $n_{\mathrm{d}}$. The corresponding proper transfer function $H_{\mathrm{o}}(q)$ is assumed to be stable and to have a stable inverse. In case $C_{\mathrm{o}}\left(q^{-1}\right)=D_{\mathrm{o}}\left(q^{-1}\right)=1,(3)$ defines an OE noise model, however with $C_{\mathrm{o}}\left(q^{-1}\right) \neq D_{\mathrm{o}}\left(q^{-1}\right),(3)$ is general enough to represent BJ-type of noise models.

\footnotetext{
$1 f: \mathbb{R}^{n} \rightarrow \mathbb{R}$ is a real meromorphic function if $f=g / h$ with $g, h$ analytic and $h \neq 0$.
}

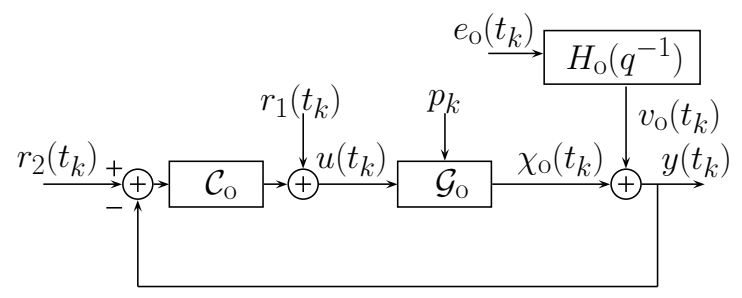

Fig. 1. Closed-loop LPV system

In terms of the closed-loop setting of Fig. 1, $u\left(t_{k}\right)=$ $r_{1}\left(t_{k}\right)+\mathcal{C}_{\mathrm{o}}\left(r_{2}\left(t_{k}\right)-y\left(t_{k}\right)\right)$, where $\mathcal{C}_{\mathrm{o}}$ is the operator form of the controller. The controller can be any LTI, nonlinear or LPV controller, under the assumptions that

- $\mathcal{C}_{\mathrm{o}}$ is a priori known;

- $\mathcal{C}_{\mathrm{O}}$ ensures BIBO stability of the closed-loop system $\mathcal{S}_{\mathrm{O}}$ for any $p \in \mathbb{P}^{\mathbb{Z}}$.

\subsection{Model considered}

Next we introduce a model structure and parameterization for the identification of $\mathcal{S}_{\mathrm{o}}$, where, according to datagenerating system, i.e. (1a-b) and (3), the noise model and the process model are parameterized separately. The proposed LPV-BJ model, denoted in the sequel as $\mathcal{M}_{\theta}$, is defined as:

$$
\begin{aligned}
A\left(p_{k}, q^{-1}, \rho\right) \chi\left(t_{k}\right) & =B\left(p_{k}, q^{-1}, \rho\right) u\left(t_{k-d}\right), \\
D\left(q^{-1}, \eta\right) v\left(t_{k}\right) & =C\left(q^{-1}, \eta\right) e\left(t_{k}\right) \\
y\left(t_{k}\right) & =\chi\left(t_{k}\right)+v\left(t_{k}\right)
\end{aligned}
$$

with parameters $\theta=\left[\rho^{\top} \eta^{\top}\right]$ and with $u\left(t_{k}\right)=r_{1}\left(t_{k}\right)+$ $\mathcal{C}_{\mathrm{o}}\left(r_{2}\left(t_{k}\right)-y\left(t_{k}\right)\right)$. The process model part of $\mathcal{M}_{\theta}$, denoted by $\mathcal{G}_{\rho}$, is defined in terms of the LPV-IO representation (4a) where $A$ and $B$ are polynomials with order $n_{\mathrm{a}}$ and $n_{\mathrm{b}}$ respectively and with $p$-dependent coefficients $a_{i}$ and $b_{j}$ parameterized as

$$
\begin{aligned}
& a_{i}\left(p_{k}\right)=a_{i, 0}+\sum_{l=1}^{n_{\alpha}} a_{i, l} f_{l}\left(p_{k}\right) \quad i=1, \ldots, n_{\mathrm{a}} \\
& b_{j}\left(p_{k}\right)=b_{j, 0}+\sum_{l=1}^{n_{\beta}} b_{j, l} g_{l}\left(p_{k}\right) \quad j=0, \ldots, n_{\mathrm{b}}
\end{aligned}
$$

In this parametrization, $\left\{f_{l}\right\}_{l=1}^{n_{\alpha}}$ and $\left\{g_{l}\right\}_{l=1}^{n_{\beta}}$ are a priori chosen meromorphic functions of $p$, with static dependence, allowing the identifiability of the model (linearly independent functions on $\mathbb{P}$ for example). The associated model parameters $\rho$ are stacked column-wise:

$$
\rho=\left[\begin{array}{llllll}
\mathrm{a}_{1} & \ldots & \mathrm{a}_{n_{\mathrm{a}}} & \mathrm{b}_{0} & \ldots & \mathrm{b}_{n_{\mathrm{b}}}
\end{array}\right]^{\top} \in \Theta_{\rho} \subset \mathbb{R}^{n_{\rho}},
$$

where $\mathrm{a}_{i}=\left[\begin{array}{llll}a_{i, 0} & a_{i, 1} & \ldots & a_{i, n_{\alpha}}\end{array}\right], \mathrm{b}_{j}=\left[\begin{array}{lllll}b_{j, 0} & b_{j, 1} & \ldots & b_{j, n_{\beta}}\end{array}\right]$, $n_{\rho}=n_{\mathrm{a}}\left(n_{\alpha}+1\right)+\left(n_{\mathrm{b}}+1\right)\left(n_{\beta}+1\right)$ and $\Theta{ }_{\rho} \subset \mathbb{R}^{n_{\rho}}$ is such that (4a) is BIBO stable for all $\rho \in \Theta_{\rho}$ and $p \in \mathbb{P}^{\mathbb{Z}}$.

The noise-model part of $\mathcal{M}_{\theta}$, denoted by $\mathcal{H}_{\eta}$, is defined in terms of (4b) where $C$ and $D$ are monic polynomials with order $n_{\mathrm{c}}$ and $n_{\mathrm{d}}$ respectively and with constant coefficients $c_{i}$ and $d_{j}$. These model parameters are stacked columnwise in the parameter vector,

$$
\eta=\left[\begin{array}{llllll}
c_{1} & \ldots & c_{n_{\mathrm{c}}} & d_{1} & \ldots & d_{n_{\mathrm{d}}}
\end{array}\right]^{\top} \in \Theta_{\eta} \subset \mathbb{R}^{n_{\eta}},
$$

where $n_{\eta}=n_{\mathrm{c}}+n_{\mathrm{d}}$. As (4b) is LTI, it can be represented by a transfer function $H(q, \eta)=\frac{C\left(q^{-1}, \eta\right)}{D\left(q^{-1}, \eta\right)}$. It is further 
assumed that $\eta \in \Theta_{\eta} \subset \mathbb{R}^{n_{\eta}}$ such that $H(z, \eta)$ is stable (analytic in the exterior of the unit circle) and has a stable inverse.

Introduce also $\mathcal{G}=\left\{\mathcal{G}_{\rho} \mid \rho \in \Theta_{\rho}\right\}$ and $\mathcal{H}=\left\{\mathcal{H}_{\eta} \mid \eta \in \Theta_{\eta}\right\}$, as the collection of all process and noise models in the form of (4a) and (4b). Then the model set, denoted as $\mathcal{M}$, takes the form

$$
\mathcal{M}=\left\{\left(\mathcal{G}_{\rho}, \mathcal{H}_{\eta}\right) \mid \operatorname{col}(\rho, \eta)=\theta \in \Theta_{\rho} \times \Theta_{\eta}\right\},
$$

corresponding to the set of candidate models in which we seek the model that explains data gathered from $\mathcal{S}_{\mathrm{o}}$ the best under a given identification criterion.

\subsection{Identification problem statement}

Denote $\mathcal{D}_{N}=\left\{y\left(t_{k}\right), u\left(t_{k}\right), p\left(t_{k}\right)\right\}_{k=1}^{N}$ a data sequence of $\mathcal{S}_{\text {o }}$. In Laurain et al. (2010), it has been shown, that a onestep-ahead output predictor $\hat{y}_{\theta}\left(t_{k} \mid t_{k-1}\right)$ can be formulated w.r.t. the considered model structure (4a-c) under the commonly used assumption that noise-free observation of the sequence $\left\{p_{k}, p_{k-1}, \ldots\right\}$ is available. Recently it has been proved that using estimated moments, such a predictor can be formulated if $p$ is observed up to an additive white noise independent from $v_{\mathrm{o}}$, see Tóth et al. (2011), however for the sake of simplicity we will only consider the previous case. These results allow to formulate (in either case) the estimation of $\theta$, as the minimization of the one-step-ahead prediction error: $\varepsilon_{\theta}=y\left(t_{k}\right)-$ $\hat{y}_{\theta}\left(t_{k} \mid t_{k-1}\right)$. This minimization is formulated in terms of an identification criterion $W\left(\mathcal{D}_{N}, \theta\right)$, like the least squares criterion

$$
W\left(\mathcal{D}_{N}, \theta\right)=\frac{1}{N} \sum_{k=1}^{N} \varepsilon_{\theta}^{2}\left(t_{k}\right)=\frac{1}{N}\left\|\varepsilon_{\theta}\left(t_{k}\right)\right\|_{\ell_{2}}^{2},
$$

such that the parameter estimate is

$$
\hat{\theta}_{N}=\arg \min _{\theta \in \Theta_{\rho} \times \Theta_{\eta}} W\left(\mathcal{D}_{N}, \theta\right) .
$$

Based on the previous considerations, the identification problem addressed in the sequel is defined as follows:

Problem 1. Given a discrete-time LPV data-generating system $\mathcal{S}_{\mathrm{O}}$ in the closed-loop setting of Fig. 1 with a priori known stabilizing controller $\mathcal{C}_{\mathrm{o}}$. Based on the LPV-BJ model structure $\mathcal{M}_{\theta}$ defined by (4a-c) and a data set $\mathcal{D}_{N}$ collected from $\mathcal{S}_{\mathrm{o}}$, estimate the parameter vector $\theta$ as the minimization of (9) under the following assumptions:

A1 $\mathcal{S}_{\mathrm{o}} \in \mathcal{M}$.

A2 In the parametrization (5a-b) of $A$ and $B,\left\{f_{l}\right\}_{l=1}^{n_{\alpha}}$ and $\left\{g_{l}\right\}_{l=1}^{n_{\beta}}$ are chosen such that $\mathcal{M}_{\theta}$ is identifiable.

A3 $\mathcal{D}_{N}$ is informative w.r.t. $\mathcal{M}_{\theta}$, i.e. if $\theta_{1} \neq \theta_{2}$ then $\mathcal{M}_{\theta_{1}}$ and $\mathcal{M}_{\theta_{2}}$ lead to different prediction errors given $\mathcal{D}_{N}$.

A4 $\mathcal{S}_{\mathrm{O}}$ is BIBO stable, i.e. for any bounded $p \in \mathbb{P}^{\mathbb{Z}}$ and $u \in \mathbb{R}^{\mathbb{Z}}$, the output of $\mathcal{S}_{\mathrm{o}}$ is bounded Tóth (2010).

A5 The closed-loop system with the controller $\mathcal{C}_{\mathrm{o}}$ and model $\mathcal{M}_{\theta}$ is BIBO stable for any $\theta \in \Theta_{\rho} \times \Theta_{\eta}$, i.e. the controller stabilizes $\mathcal{S}_{\mathrm{o}}$ and also any model in the considered model set.

A6 $p$ is treated as an external variable of the system.

In Laurain et al. (2010), a refined instrumental variable (RIV) based approach has been introduced to solve the estimation problem of $\mathcal{M}_{\theta}$ in an open-loop setting. However, a major difference between the open-loop setting considered in Laurain et al. (2010) and the closed-loop identification setting of Problem 1 is the correlation of the signal $u$ with the noise $e_{\mathrm{o}}$ due to the feedback loop. Thus in the next section, we focus on the problem how to determine a suitable instrument that allows the extension of the LPV-RIV approach to the closed-loop case.

\section{REFORMULATION OF THE IDENTIFICATION PROBLEM}

In Laurain et al. (2010) it has been shown that reformulation of (4a-c) as a multiple-input single-output (MISO) LTI model allows an elegant solution of the filtering problem associated with LPV-IV approaches. This reformulation is necessary to avoid the problem of non-commutativity of parameter-varying filters and hence required to solve Problem 1. Consequently, (4a-c) are rewritten as

$$
\begin{aligned}
\underbrace{\chi\left(t_{k}\right)+\sum_{i=1}^{n_{\mathrm{a}}} a_{i, 0} \chi\left(t_{k-i}\right)}_{F\left(q^{-1}\right) \chi\left(t_{k}\right)} & +\sum_{i=1}^{n_{\mathrm{a}}} \sum_{l=1}^{n_{\alpha}} a_{i, l} \underbrace{f_{l}\left(p_{k}\right) \chi\left(t_{k-i}\right)}_{\chi_{i, l}\left(t_{k}\right)} \\
= & \sum_{j=0}^{n_{\mathrm{b}}} \sum_{l=0}^{n_{\beta}} b_{j, l} \underbrace{g_{l}\left(p_{k}\right) u\left(t_{k-d-j}\right)}_{u_{j, l}\left(t_{k}\right)}) \\
v\left(t_{k}\right) & =\frac{C\left(q^{-1}, \eta\right)}{D\left(q^{-1}, \eta\right)} e\left(t_{k}\right) \\
y\left(t_{k}\right) & =\chi\left(t_{k}\right)+v\left(t_{k}\right)
\end{aligned}
$$

where $F\left(q^{-1}\right)=1+\sum_{i=1}^{n_{\mathrm{a}}} a_{i, 0} q^{-i}$ and $g_{0}(\cdot)=1$. Note that in this way, the LPV-BJ model is rewritten as a MISO system with $\left(n_{\mathrm{b}}+1\right)\left(n_{\beta}+1\right)+n_{\mathrm{a}} n_{\alpha}$ inputs $\left\{\chi_{i, l}\right\}_{i=1, l=1}^{n_{\mathrm{a}}, n_{\alpha}}$ and $\left\{u_{j, l}\right\}_{j=0, l=0}^{n_{\mathrm{b}}, n_{\beta}} . F\left(q^{-1}\right)$ does not depend on $p_{k}$, and (11a) can be rewritten as the following LTI model:

$$
\begin{aligned}
y\left(t_{k}\right)=- & \sum_{i=1}^{n_{\mathrm{a}}} \sum_{l=1}^{n_{\alpha}} \frac{a_{i, l}}{F\left(q^{-1}\right)} \chi_{i, l}\left(t_{k}\right) \\
& +\sum_{j=0}^{n_{\mathrm{b}}} \sum_{l=0}^{n_{\beta}} \frac{b_{j, l}}{F\left(q^{-1}\right)} u_{k, j}\left(t_{k}\right)+H(q) e\left(t_{k}\right),
\end{aligned}
$$

Note that (12) is an equivalent form of (4a-c), but it is not a representation of the associated LPV system as it includes lumped output variables in the signals $\left\{\chi_{i, l}\right\}_{i=1, l=1}^{n_{\mathrm{a}}, n_{\alpha}}$.

\section{RIV APPROACH FOR CLOSED-LOOP LPV SYSTEMS}

Using the MISO-LTI formulation (12), the estimation problem of the parameters can be formulated as a linear regression which allows optimal solution of (9) w.r.t. LPVBJ models. To achieve this solution, one possible way is to develop an extension of the refined instrumental variable (RIV) approach of the closed-loop LTI identification framework. Next we derive this extension, which, as we will see, provides an easily implementable iterative estimation scheme.

\subsection{The refined instrumental variable estimate}

Using (12), $y\left(t_{k}\right)$ can be written in a linear regression form:

$$
y\left(t_{k}\right)=\varphi^{\top}\left(t_{k}\right) \rho+\tilde{v}\left(t_{k}\right),
$$

where $\tilde{v}\left(t_{k}\right)=F\left(q^{-1}, \rho\right) v\left(t_{k}\right)$ and 


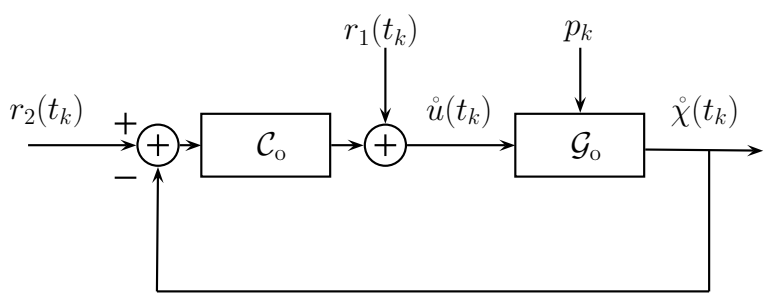

Fig. 2. Auxiliary model

$$
\begin{aligned}
& \varphi\left(t_{k}\right)=\left[-y\left(t_{k-1}\right) \ldots-y\left(t_{k-n_{\mathrm{a}}}\right)-\chi_{1,1}\left(t_{k}\right) \ldots\right. \\
& \left.-\chi_{n_{\mathrm{a}}, n_{\alpha}}\left(t_{k}\right) u_{0,0}\left(t_{k}\right) \ldots u_{n_{\mathrm{b}}, n_{\beta}}\left(t_{k}\right)\right]^{\top},
\end{aligned}
$$

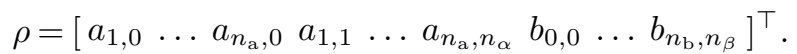

Two difficulties remain to obtain the minimum of (9) based on (13): the regressor $\varphi\left(t_{k}\right)$ contains unknown terms $\left\{\chi_{i, l}\left(t_{k}\right)\right\}_{i=1, l=0}^{n_{\mathrm{a}}, n_{\alpha}}$ and all of its elements are corrupted with the colored noise $v\left(t_{k}\right)$. To resolve this problem, an appropriate instrumental variable $\zeta\left(t_{k}\right)$ can be introduced such that the (extended-IV) estimate of $\rho$ can be given as Söderström and Stoica (1983):

$$
\begin{gathered}
\hat{\rho}_{\mathrm{XIV}}(N)=\arg \min _{\rho \in \mathbb{R}^{n} \rho} \|\left[\frac{1}{N} \sum_{k=1}^{N} L(q) \zeta\left(t_{k}\right) L(q) \varphi^{\top}\left(t_{k}\right)\right] \rho \\
-\left[\frac{1}{N} \sum_{t=1}^{N} L(q) \zeta\left(t_{k}\right) L(q) y\left(t_{k}\right)\right] \|_{W}^{2}, \quad(14)
\end{gathered}
$$

where $\|x\|_{W}^{2}=x^{T} W x$, with $W$ a positive definite weighting matrix and $L(q)$ is a stable prefilter. If $G_{\mathrm{o}} \in \mathcal{G}$, the estimate (14) is consistent under the following well-known conditions $^{2}$ :

C1 $\overline{\mathbb{E}}\left\{L(q) \zeta\left(t_{k}\right) L(q) \varphi^{\top}\left(t_{k}\right)\right\}$ is full column rank.

C2 $\overline{\mathbb{E}}\left\{L(q) \zeta\left(t_{k}\right) L(q) \tilde{v}\left(t_{k}\right)\right\}=0$.

Moreover it has been shown in Söderström and Stoica (1983) and Young (1984) that in general for IV methods, based on (14), the minimum variance estimator can be achieved if:

\section{C3 $W=I$.}

C4 $\zeta\left(t_{k}\right)$ is chosen as the noise-free version of (13) and is therefore defined in the present LPV case as:

$$
\begin{aligned}
& \zeta\left(t_{k}\right)=\left[-\stackrel{\circ}{\chi}\left(t_{k-1}\right) \ldots-\dot{\chi}\left(t_{k-n_{\mathrm{a}}}\right)-\dot{\circ}_{1,1}\left(t_{k}\right) \ldots\right. \\
& \left.-\stackrel{\circ}{\chi}_{n_{\mathrm{a}}, n_{\alpha}}\left(t_{k}\right) \stackrel{\circ}{u}_{0,0}\left(t_{k}\right) \ldots{\stackrel{\circ}{n_{\mathrm{b}}, n_{\beta}}}\left(t_{k}\right)\right]^{\top}
\end{aligned}
$$

where $\stackrel{i}{ }$ and $\dot{\chi}$ are the signals from the auxiliary system as presented in Fig. 2 and where the index notations are the same as presented in (12).

C5 $\mathcal{G}_{\mathrm{o}} \in \mathcal{G}$ and $n_{\rho}$ is equal to the minimal number of parameters required to represent $\mathcal{G}_{\mathrm{o}}$ with the considered model structure.

C6 $L(q)$ is chosen as in Laurain et al. (2010):

$$
L(q)=\frac{D\left(q^{-1}\right)}{F\left(q^{-1}\right) C\left(q^{-1}\right)} .
$$

Full column rank of $\overline{\mathbb{E}}\left\{L(q) \varphi\left(t_{k}\right) L(q) \varphi^{\top}\left(t_{k}\right)\right\}$ is ensured by the informativity of the data set (A3). Note that conditions about informativity w.r.t. LPV-BJ model structures is in the focus of current research (see Bamieh and Giarré

2 The notation $\overline{\mathbb{E}}\{\}=.\lim _{N \rightarrow \infty} \frac{1}{N} \sum_{t=1}^{N} \mathbb{E}\{$.$\} is adopted from the$ prediction error framework of Ljung (1999).
(2002); Tóth (2010); Wei and Del Re (2006) for results in the ARX case). To fulfill C1 under A3, the discussion can be found in Söderström and Stoica (1983). It is also important to note that in a practical situation none of $F\left(q^{-1}, \rho\right)$, $C\left(q^{-1}, \eta\right), D\left(q^{-1}, \eta\right)$ or $\left\{a_{i, l}(\rho)\right\}_{i=1, l=0}^{n_{\mathrm{a}}, n_{\alpha}},\left\{b_{j, l}(\rho)\right\}_{j=0, l=0}^{n_{\mathrm{b}}, n_{\beta}}$ is known a priori. Therefore, the RIV estimation normally involves an iterative (or relaxation) algorithm in which, at each iteration, an 'auxiliary model' is used to generate the instrumental variables (which guarantees C2), as well as the associated prefilters. Convergence of such an iterative RIV algorithm has not been proved so far and is only empirically assumed Young (2008). Furthermore, the considered LPV model can be reformulated in a LTI-MISO form only under the condition that the noise-free output terms are a priori known. Therefore, even if the presented method considerably lowers the variance in the estimated parameters, the optimality cannot be guaranteed.

\subsection{Iterative closed-loop LPV-RIV Algorithm}

Based on the previous considerations, the iterative scheme of the RIV algorithm can be extended to the closed-loop LPV case as follows.

Algorithm 1. (LPV-RIV).

Step 1 Assume that as an initialization, an ARX estimate of $\mathcal{M}_{\theta}$ is available by the LS approach, i.e. $\hat{\theta}^{(0)}=$ $\left[\left(\hat{\rho}^{(0)}\right)^{\top}\left(\hat{\eta}^{(0)}\right)^{\top}\right]^{\top}$ is given. Set $\tau=0$.

Step 2 Compute an estimate of $\chi\left(t_{k}\right)$ via

$$
A\left(p_{k}, q^{-1}, \hat{\rho}^{(\tau)}\right) \hat{\chi}\left(t_{k}\right)=B\left(p_{k}, q^{-1}, \hat{\rho}^{(\tau)}\right) u\left(t_{k-d}\right),
$$

where $\hat{\rho}^{(\tau)}$ is estimated in the previous iteration and it is assumed to be stable in analog with A5. Based on $\mathcal{M}_{\hat{\theta}(\tau)}$, deduce $\left\{\hat{\chi}_{i, l}\left(t_{k}\right)\right\}_{i=1, l=0}^{n_{\mathrm{a}, n_{\alpha}}}$ as given in (11a). It is important to note that $\hat{\chi}\left(t_{k}\right)$ is correlated with the noise as it is simulated using the measured signal $u$.

Step 3 Compute the estimated filter:

$$
L\left(q^{-1}, \hat{\theta}^{(\tau)}\right)=\frac{D\left(q^{-1}, \hat{\eta}^{(\tau)}\right)}{C\left(q^{-1}, \hat{\eta}^{(\tau)}\right) F\left(q^{-1}, \hat{\rho}^{(\tau)}\right)}
$$

and the associated filtered signals $\left\{u_{j, l}^{\mathrm{f}}\left(t_{k}\right)\right\}_{j=0, l=0}^{n_{\mathrm{b}}, n_{\beta}}$, $y_{\mathrm{f}}\left(t_{k}\right)$ and $\left\{\hat{\chi}_{i, l}^{\mathrm{f}}\left(t_{k}\right)\right\}_{i=1, l=0}^{n_{\mathrm{a}}, n_{\alpha}}$.

Step 4 Build the noisy filtered estimated regressor $\hat{\varphi}_{\mathrm{f}}\left(t_{k}\right)$ as:

$$
\begin{gathered}
\hat{\varphi}_{\mathrm{f}}\left(t_{k}\right)=\left[-y_{\mathrm{f}}\left(t_{k-1}\right) \ldots-y_{\mathrm{f}}\left(t_{k-n_{\mathrm{a}}}\right)-\hat{\chi}_{1,1}^{\mathrm{f}}\left(t_{k}\right)\right. \\
\left.\ldots-\hat{\chi}_{n_{\mathrm{a}}, n_{\alpha}}^{\mathrm{f}}\left(t_{k}\right) u_{0,0}^{\mathrm{f}}\left(t_{k}\right) \ldots u_{n_{\mathrm{b}}, n_{\beta}}^{\mathrm{f}}\left(t_{k}\right)\right]^{\top}
\end{gathered}
$$

and compute the filtered instrument $\hat{\zeta}_{\mathrm{f}}\left(t_{k}\right)$ by simulating the estimated auxiliary model according to Fig. 2:

$$
\begin{aligned}
& \hat{\zeta}_{\mathrm{f}}\left(t_{k}\right)=\left[-\hat{\dot{\chi}}_{\mathrm{f}}\left(t_{k-1}\right) \ldots-\hat{\dot{\chi}}_{\mathrm{f}}\left(t_{k-n_{\mathrm{a}}}\right)-\hat{\grave{\chi}}_{1,1}^{\mathrm{f}}\left(t_{k}\right)\right. \\
& \left.\ldots-\hat{\hat{\chi}}_{n_{\mathrm{a}}, n_{\alpha}}^{\mathrm{f}}\left(t_{k}\right) \hat{\hat{u}}_{0,0}^{\mathrm{f}}\left(t_{k}\right) \ldots \hat{\dot{u}}_{n_{\mathrm{b}}, n_{\beta}}^{\mathrm{f}}\left(t_{k}\right)\right]^{\top}
\end{aligned}
$$

Note that this instrument fulfills $\mathrm{C} 1$ and $\mathrm{C} 2$.

Step 5 The IV optimization problem can now be stated in the form

$$
\begin{aligned}
\hat{\rho}^{(\tau+1)}(N)=\arg \min _{\rho \in \mathbb{R}^{n} \rho} \| & {\left[\frac{1}{N} \sum_{k=1}^{N} \hat{\zeta}_{\mathrm{f}}\left(t_{k}\right) \hat{\varphi}_{\mathrm{f}}^{\top}\left(t_{k}\right)\right] \rho } \\
& -\left[\frac{1}{N} \sum_{k=1}^{N} \hat{\zeta}_{\mathrm{f}}\left(t_{k}\right) y_{\mathrm{f}}\left(t_{k}\right)\right] \|^{2}
\end{aligned}
$$


where the solution is obtained as

$$
\hat{\rho}^{(\tau+1)}(N)=\left[\sum_{k=1}^{N} \hat{\zeta}_{\mathrm{f}}\left(t_{k}\right) \hat{\varphi}_{\mathrm{f}}^{\top}\left(t_{k}\right)\right]^{-1} \sum_{k=1}^{N} \hat{\zeta}_{\mathrm{f}}\left(t_{k}\right) y_{\mathrm{f}}\left(t_{k}\right) .
$$

The resulting $\hat{\rho}^{(\tau+1)}(N)$ is the IV estimate of the process model associated parameter vector at iteration $\tau+1$ based on the prefiltered input/output data.

Step 6 An estimate of the noise signal $v$ is obtained as

$$
\hat{v}\left(t_{k}\right)=y\left(t_{k}\right)-\hat{\chi}\left(t_{k}, \hat{\rho}^{(\tau)}\right) .
$$

Based on $\hat{v}$, the estimation of the noise model parameter vector $\hat{\eta}^{(\tau+1)}$ follows, using e.g. ARMA estimation algorithm of the MATLAB identification toolbox (an IV approach can also be used for this purpose, see Young $(2008))$.

Step 7 If $\theta^{(\tau+1)}$ has converged or the maximum number of iterations is reached, then stop, else increase $\tau$ by 1 and go to Step 2.

Based on a similar concept, the so-called simplified LPVRIV (LPV-SRIV) method, can also be developed for the estimation of LPV-OE models. This method is based on a model structure (4a-c) with $C\left(q^{-1}, \eta\right)=D\left(q^{-1}, \eta\right)=1$ and consequently, Step 6 of Algorithm 1 can be skipped.

\section{SIMULATION EXAMPLE}

Next, the performance of the proposed RIV method with its SRIV variant are compared via a simulation example. The considered data-generating system is described as

$$
\mathcal{S}_{\mathrm{o}}\left\{\begin{array}{l}
A_{\mathrm{o}}\left(q, p_{k}\right)=1+a_{1}^{\mathrm{o}}\left(p_{k}\right) q^{-1}+a_{2}^{\mathrm{o}}\left(p_{k}\right) q^{-2} \\
B_{\mathrm{o}}\left(q, p_{k}\right)=b_{0}^{\mathrm{o}}\left(p_{k}\right) q^{-1}+b_{1}^{\mathrm{o}}\left(p_{k}\right) q^{-2} \\
H_{\mathrm{o}}(q)=\frac{1}{1-q^{-1}+0.2 q^{-2}}
\end{array}\right.
$$

where $v_{\mathrm{o}}\left(t_{k}\right)=H_{\mathrm{o}}(q) e_{\mathrm{o}}\left(t_{k}\right)$ and

$$
\begin{aligned}
& a_{1}^{\mathrm{o}}\left(p_{k}\right)=1-0.5 p_{k}-0.1 p_{k}^{2}, \\
& a_{2}^{\mathrm{o}}\left(p_{k}\right)=0.5-0.7 p_{k}-0.1 p_{k}^{2}, \\
& b_{0}^{\mathrm{o}}\left(p_{k}\right)=0.5-0.4 p_{k}+0.01 p_{k}^{2}, \\
& b_{1}^{\mathrm{o}}\left(p_{k}\right)=0.2-0.3 p_{k}-0.02 p_{k}^{2} .
\end{aligned}
$$

According to Fig. $1, \mathcal{S}_{\mathrm{o}}$ is considered with a stabilizing feedback controller given in this case as

$$
u\left(t_{k}\right)=\frac{1+0.5 q^{-1}}{1-0.85 q^{-1}}\left(r_{2}\left(t_{k}\right)-y\left(t_{k}\right)\right)+r_{1}\left(t_{k}\right),
$$

To generate data sets $\mathcal{D}_{N}$ of $\mathcal{S}_{\mathrm{o}}$ for identification and validation, the closed-loop system is simulated using $p_{k}=$ $0.5 \sin (0.35 \pi k)+0.5$ and $r_{1}\left(t_{k}\right)$ taken as a white noise with a uniform distribution $\mathcal{U}(-1,1)$ and with length $N=4000$. In the simulation, the white noise disturbance $e_{\mathrm{o}}\left(t_{k}\right) \in \mathcal{N}\left(0, \sigma^{2}\right)$ is considered with different $\sigma^{2}>0$ to investigate low and high noise conditions.

In the sequel, the conventional LPV Least Square (LS) method Bamieh and Giarré (2002) is compared to the proposed IV approaches. The least square method is not suited for OE models and may not be a good candidate for comparison. Nonetheless, this method is known to result in parameter estimates with low variance and is therefore a good indicator for the efficiency of the RIV estimator. In order to the demonstrate the efficiency of the proposed
RIV estimators without effect of under-modeling and overparametrization, full structural knowledge about system is assumed. Thus the proposed LPV Closed Loop Refined Instrumental Variable method (LPV-CLRIV) is considered with the following LPV-BJ model:

$\mathcal{M}_{\theta}^{\mathrm{LPV}-\mathrm{RIV}}\left\{\begin{array}{l}A\left(p_{k}, q^{-1}, \rho\right)=1+a_{1}\left(p_{k}\right) q^{-1}+a_{2}\left(p_{k}\right) q^{-2} \\ B\left(p_{k}, q^{-1}, \rho\right)=b_{0}\left(p_{k}\right) q^{-1}+b_{1}\left(p_{k}\right) q^{-2} \\ H\left(p_{k}, q, \eta\right)=\frac{1}{1+d_{1} q^{-1}+d_{2} q^{-2}}\end{array}\right.$

which represents the situation $\mathcal{S}_{\mathrm{o}} \in \mathcal{M}$. In analog, the LPV Simplified Closed-Loop Refined Instrumental Variable approach (LPV-SCLRIV) is used with the following LPVOE model:

$\mathcal{M}_{\theta}^{\mathrm{LPV}-\operatorname{SRIV}}\left\{\begin{array}{l}A\left(p_{k}, q^{-1}, \rho\right)=1+a_{1}\left(p_{k}\right) q^{-1}+a_{2}\left(p_{k}\right) q^{-2} \\ B\left(p_{k}, q^{-1}, \rho\right)=b_{0}\left(p_{k}\right) q^{-1}+b_{1}\left(p_{k}\right) q^{-2} \\ H\left(p_{k}, q, \eta\right)=1\end{array}\right.$

which represents the case when $\mathcal{G}_{\mathrm{o}} \in \mathcal{G}, \mathcal{H}_{\mathrm{o}} \notin \mathcal{H}$. In both cases, full parametrization of the associated coefficient functions is considered:

$$
\begin{aligned}
& a_{1}\left(p_{k}\right)=a_{1,0}+a_{1,1} p_{k}+a_{1,2} p_{k}^{2} \\
& a_{2}\left(p_{k}\right)=a_{2,0}+a_{2,1} p_{k}+a_{2,2} p_{k}^{2} \\
& b_{0}\left(p_{k}\right)=b_{0,0}+b_{0,1} p_{k}+b_{0,2} p_{k}^{2} \\
& b_{1}\left(p_{k}\right)=b_{1,0}+b_{1,1} p_{k}+b_{1,2} p_{k}^{2}
\end{aligned}
$$

To provide representative results, a Monte-Carlo simulation of $N_{\mathrm{MC}}=100$ runs is accomplished at different noise levels: $20 \mathrm{~dB}, 15 \mathrm{~dB}, 10 \mathrm{~dB}$ and $5 \mathrm{~dB}$. In Table 1 , the norm of the bias $(\mathrm{BN})\left\|\rho_{o}-\overline{\mathbb{E}}(\hat{\rho})\right\|_{\ell_{2}}$ and variance $(\mathrm{VN})\|\overline{\mathbb{E}}(\hat{\rho}-\overline{\mathbb{E}}(\hat{\rho}))\|_{\ell_{2}}$ norms of the resulting estimated parameter vectors are given, where $\overline{\mathbb{E}}$ is the mean operator over the Monte-Carlo simulation. The number of iterations (Nit) needed for convergence is also given. Table 1 shows that contrary to the LS method the IV methods are unbiased which is in line with the theoretical results. For SNR down to $5 \mathrm{~dB}$, the LPV-CLRIV produces a parameter variance very close to the one obtained with the LS method. Although the statistical optimality of the algorithm cannot be proved, this latter result shows on this example, that the LPV-CLRIV algorithm results in accurate estimates. The suboptimal LPV-CLSRIV method offers satisfying results, considering that the used noise model cannot capture the dynamics of $v_{\mathrm{o}}$. The variance in the estimated parameters is twice as much as in the LPV-CLRIV case but it is still close to the variance of the LS method.

Table $1 . \ell_{2}$-norm of the bias and variance of the estimated parameters at different SNR levels.

\begin{tabular}{|c||c||c|c|c|c|}
\hline Method & & $20 \mathrm{~dB}$ & $15 \mathrm{~dB}$ & $10 \mathrm{~dB}$ & $5 \mathrm{~dB}$ \\
\hline \multirow{2}{*}{ LS } & BN & 1.5736 & 2.3922 & 2.0812 & 2.0908 \\
\cline { 2 - 6 } & VN & 0.0092 & 0.0140 & 0.0253 & 0.0326 \\
\hline \hline \multirow{2}{*}{ LPV } & BN & 0.0067 & 0.0145 & 0.0125 & 0.0052 \\
\cline { 2 - 6 } -SRIV & VN & 0.0011 & 0.0035 & 0.0110 & 0.0319 \\
\cline { 2 - 6 } & Nit & 16 & 16 & 17 & 19 \\
\hline \hline \multirow{2}{*}{ LPV } & BN & 0.0060 & 0.0139 & 0.0185 & 0.0146 \\
\cline { 2 - 6 }- RIV & VN & 0.000623 & 0.0018 & 0.0068 & 0.0206 \\
\cline { 2 - 6 } & Nit & 19 & 19 & 20 & 22 \\
\hline
\end{tabular}




\section{CONCLUSION}

In this paper, the extension of an LPV-RIV approach has been introduced to provide an efficient solution for the closed-loop identification of LPV systems with BoxJenkins type of noise models. The approach has been formulated with the assumption of prior knowledge of the controller but without any restriction on its structure like LTI, LPV, nonlinear, etc. It has been shown that under given conditions the proposed approach provides consistent estimates and its performance has been demonstrated on a representative example including a comparison to the least-squares approach. The presented example has shown that the proposed procedure is robust to noise and that the obtained estimates are accurate.

\section{REFERENCES}

H. Abbas and H. Werner. An instrumental variable technique for open-loop and closed-loop identification of input-output LPV models. In Proc. of the European Control Conf., pages 2646-2651, Budapest, Hungary, 2009.

B. Bamieh and L. Giarré. Identification of linear parameter varying models. Int. Journal of Robust and Nonlinear Control, 12:841-853, 2002.

S. Boonto and H. Werner. Closed-loop system identification of LPV input-output models - application to an arm-driven pendulum. In Proc. of the 47th IEEE Conf. on Decision and Control, pages 2606-2611, Cancun, Mexico, Dec. 2008.

M. Butcher, A. Karimi, and R. Longchamp. On the consistency of certain identification methods for linear parameter varying systems. In Proc. of the 17th IFAC World Congress, pages 4018-4023, Seoul, Korea, July 2008.

F. Casella and M. Lovera. LPV/LFT modelling and identification: overview, synergies and a case study. In IEEE International Symposium on Computer-Aided Control System Design, pages 852-857, San Antonio, Texas, USA, Sept. 2008.

U. Forssell and L. Ljung. Closed-loop identification revisited. Automatica, 35(7):1215-1241, 1999.

I. D. Landau and A. Karimi. Recursive algorithms for identification in closed loop: A unified approach and evaluation. Automatica, 33(8):1499-1523, 1997.

V. Laurain, M. Gilson, R. Tóth, and H. Garnier. Refined instrumental variable methods for identification of LPV Box-Jenkins models. Automatica, 46(6):959-967, 2010.

L. Ljung. System Identification, theory for the user. Prentice Hall, 1999.

A. Packard. Gain scheduling via linear fractional transformations. Systems \&6 Control Letters, 22(2):79-92, 1994.

C. W. Scherer. Mixed $\mathcal{H}_{2} / \mathcal{H}_{\infty}$ control for time-varying and linear parametrically-varying systems. Int. Journal of Robust and Nonlinear Control, 6(9-10):929-952, 1996.

T. Söderström and P. Stoica. Instrumental Variable Methods for System Identification. Springer-Verlag, New York, 1983.

R. Tóth. Modeling and Identification of Linear ParameterVarying Systems. Lecture Notes in Control and Information Sciences, Vol. 403. Springer-Germany, 2010.

R. Tóth, E. Bitar, P. S. C. Heuberger, P. M. J. Van den Hof, and K. Poolla. A prediction-error identification framework for linear parameter-varying systems. Submitted to Automatica, 2011.

P. M. J. Van den Hof. Closed-loop issues in system identification. Annual reviews in control, 22:173-186, 1998.

J. W. van Wingerden and M. Verhaegen. Subspace identification of bilinear and LPV systems for open- and closed-loop data. Automatica, 45(2):372-381, 2009.

X. Wei and L. Del Re. On persistent excitation for parameter estimation of quasi-LPV systems and its application in modeling of diesel engine torque. In Proc. of the 14th IFAC Symposium on System Identification, pages 517-522, Newcastle, Australia, Mar. 2006.

P. C. Young. Recursive Estimation and Time-Series Analysis. Springer-Verlag, Berlin, 1984.

P. C. Young. The refined instrumental variable method: Unified estimation of discrete and continuous-time transfer function models. Journal Européen des Systèmes Automatisés, 42:149-179, 2008. 